\title{
VO: A strongly correlated metal close to a Mott-Hubbard transition
}

\author{
F. Rivadulla, ${ }^{1 *}$ J. Fernández-Rossier, ${ }^{2}$ M. García-Hernández, ${ }^{3}$ M. A. López-Quintela, ${ }^{1}$ J. Rivas, ${ }^{4}$ and J. B. Goodenough ${ }^{5}$ \\ ${ }^{1}$ Physical-Chemistry Department, University of Santiago de Compostela, 15782 Santiago de Compostela, Spain \\ ${ }^{2}$ Applied Physics Department, University of Alicante, San Vicente del Raspeig, 03690 Alicante, Spain \\ ${ }^{3}$ Instituto de Ciencia de Materiales de Madrid (CSIC), Cantoblanco, E-28049 Madrid, Spain \\ ${ }^{4}$ Applied Physics Department, University of Santiago de Compostela, 15782 Santiago de Compostela, Spain \\ ${ }^{5}$ Texas Materials Institute, ETC 9.102, The University of Texas at Austin, 1 University Station, C2201, Austin, Texas 78712, USA
}

(Received 9 May 2007; revised manuscript received 3 July 2007; published 19 November 2007)

\begin{abstract}
Here, we present experimental and computational evidences to support that rocksalt cubic VO is a strongly correlated metal with non-Fermi-liquid thermodynamics and an unusually strong spin-lattice coupling. An unexpected change of sign of metallic thermopower with composition is tentatively ascribed to the presence of a pseudogap in the density of states. These properties are discussed as signatures of the proximity to a magnetic quantum phase transition. The results are summarized in an electronic phase diagram for the $3 d$ monoxides, which resembles that of other strongly correlated systems. The structural and electronic simplicity of $3 d$ monoxides makes them ideal candidates to progress in the understanding of highly correlated electron systems.
\end{abstract}

DOI: $10.1103 /$ PhysRevB.76.205110

PACS number(s): 71.30. $+\mathrm{h}, 71.10 . \mathrm{Hf}, 71.27 .+\mathrm{a}$

\section{INTRODUCTION}

The outer $d$ electrons of most transition metals occupy narrow bands that, as long as the bandwidth $(W)$ remains larger than the intra-atomic Coulombic repulsion $(U)$, retain an itinerant-electron character described by the Landau Fermi-liquid (FL) picture. The FL theory correctly predicts that low-temperature magnetic susceptibility $\chi$, specific heat $C(T)$, and resistivity $\rho(T)$ scale as $T^{0}, T^{1}$, and $T^{2}$, respectively. ${ }^{1}$

However, for materials with $U>W$, the interatomic interactions are not strong enough to screen effectively the intraatomic interactions so that electrons become localized and the spectrum of charged excitations acquires a gap. These are the so-called Mott-Hubbard insulators. ${ }^{2}$ It has long been recognized that the collective quantum states of conductors and insulators are fundamentally different phases separated by some kind of quantum phase transition (QPT) boundary. In the neighborhood of the QPT, interactions are non-negligible and $\chi, C / T$, and $\rho(T)$ deviate from the FL scenario. This behavior has been observed in heavy fermions, ${ }^{3,4}$ cuprates, ${ }^{5}$ manganites, ${ }^{6}$ and even simple metallic alloys ${ }^{7}$ and other materials ${ }^{8}$ close to a magnetic QPT. Moreover, a depression of the density of states around the Fermi energy, the socalled pseudo-gap, has been shown to occur in many of these systems. ${ }^{9-11}$

In rocksalt transition-metal (TM) monoxides (TiO, VO, $\mathrm{MnO}, \mathrm{FeO}, \mathrm{CoO}$, and $\mathrm{NiO}$ ), octahedral-site $M-\mathrm{O}$ interactions split the $3 d$ orbitals into a more stable, threefold-degenerate manifold of $\pi$-bonding $t_{2 g}$ orbitals $(x y, y z \pm i z x)$ and a twofold-degenerate manifold of $\sigma$-bonding $e_{g}$ orbitals by an energy $\Delta_{c}$. Occupation of these bands determines the electronic properties across the series: correlation-driven insulators with antiferromagnetic order in the case of $\mathrm{MnO}, \mathrm{FeO}$, $\mathrm{CoO}$, and $\mathrm{NiO}$, and a Fermi-liquid metal, with a superconducting phase below $1 \mathrm{~K}$, in the case of TiO. ${ }^{12}$ Naively, a quantum phase transition that separates metallic $\mathrm{TiO}$ from the insulating antiferromagnet $\mathrm{MnO}$ can be accomplished as additional electrons are added into the $d$ levels of isostruc- tural TM monoxides. In the crossover region between these two antagonistic phases are located $\mathrm{CrO}$, whose bulk synthesis remains a challenge, and $\mathrm{VO}$, whose electronic properties are discussed here.

In this paper, we report experimental measurements of specific heat, electronic transport, and magnetic susceptibility of a series of samples of $\mathrm{TiO}_{x}$ and $\mathrm{VO}_{x}$, with $0.9<x$ $<1$.1. In spite of their similar electronic structure, we will present solid evidence that $\mathrm{TiO}$ is well described by conventional FL band theory while VO departs from FL picture. The results are discussed in terms of the proximity to a magnetic or electronic quantum phase transition.

\section{EXPERIMENT}

Polycrystalline $\mathrm{VO}_{x}$ and $\mathrm{TiO}_{x}$ have been generally synthesized by arc melting and casting. This method presents important experimental difficulties that have resulted in disagreement between data published before the work of Banus et al. ${ }^{13}$ To avoid these problems, we propose an alternative synthetic route that yields $\mathrm{VO}_{x}$ with controllable stoichiometry and of quality comparable to traditional methods. $\mathrm{TiO}_{x}$ and $\mathrm{VO}_{x}$ are perfectly stable in a wide compositional range, approximately between $0.8<x<1.2,{ }^{13}$ and even the stoichiometric compounds $(x=1)$ show $16 \%$ of vacancies at both the metal and oxygen sites to shorten the lattice parameter so as to increase $W .{ }^{14}$ For the synthesis of $\mathrm{VO}_{x}$, high purity vanadium metal and $\mathrm{V}_{2} \mathrm{O}_{3}$ were mixed in stoichiometric proportions, according to the desired value of $x$. The powders were ground, mixed, and pressed into pellets in an Ar atmosphere before being transferred and sealed into a silica tube that had been evacuated down to $P$ $\approx 10^{-5}-10^{-4}$ Torr. The pellet was placed in a small alumina crucible to avoid reaction of $\mathrm{V}$ with the tube, which produces traces of $\mathrm{V}_{3} \mathrm{Si}$, difficult to detect by $\mathrm{x}$ ray. The ampoules were annealed at $1100{ }^{\circ} \mathrm{C}$ for $24 \mathrm{~h}$ and quenched into an ice-water bath. Quenching from high temperature avoids problems of disproportionation, which was probably the origin of the metal-insulator transition attributed to $\mathrm{VO}$ in the 


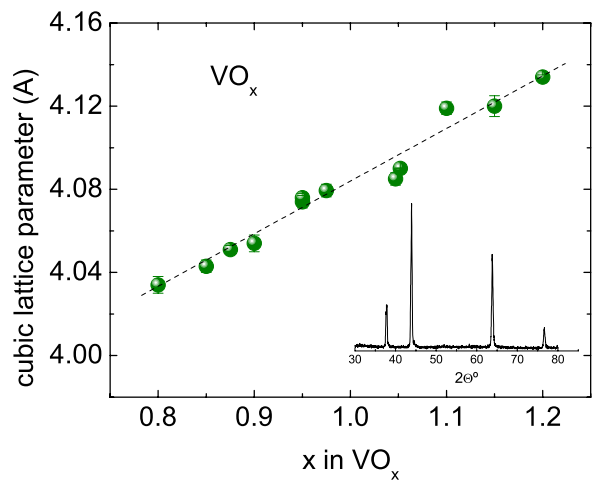

FIG. 1. (Color online) Evolution of lattice parameters in $\mathrm{VO}_{x}$ with $x$. The results are practically identical to those obtained by Banus et al. (Ref. 13), whose samples where synthesized by arc melting and casting. Inset: example of x-ray pattern, in this case, for $\mathrm{VO}_{1.05}$.

oldest literature. ${ }^{15}$ Quenched pellets were ground and cold pressed at $16 \times 10^{3} \mathrm{~kg} / \mathrm{cm}^{2}$ before being again sealed in evacuated silica tubes and refired at $1100{ }^{\circ} \mathrm{C}$ for $24 \mathrm{~h}$. After this treatment, the pellets (shining gray) were polished and the x-ray patterns showed only very narrow peaks of the single-phase cubic $(F m-3 m)$ material (Fig. 1). The oxygen or metal ratio $x$ in $\mathrm{VO}_{x}$ was determined by thermogravimetric analysis (Fig. 2). Powdered samples were calcined in oxygen at $1{ }^{\circ} \mathrm{C} / \mathrm{min}$ up to $600{ }^{\circ} \mathrm{C}$ and held for $16 \mathrm{~h}$. After this time, oxidation to $\mathrm{V}_{2} \mathrm{O}_{5}$ was complete; no further weight gain was detected on cooling, indicating complete combustion of the monoxide. The lattice parameters for different $x$ are in perfect agreement with the literature values ${ }^{13}$ (Fig. 1). Moreover, due to the correlation between lattice parameter and vacancy concentration, we can ensure a similar amount of vacancies as that reported in Ref. 13. The grain sizes in the sintered pellets determined by optical microscopy typically ranged between 5 and $20 \mu \mathrm{m}$.

Cubic $\mathrm{TiO}_{x}$ (shining gold) can be synthesized in a similar way from $\mathrm{Ti}$ and $\mathrm{TiO}_{2}$ at higher temperature. $\mathrm{TiO}$ is commercially available and no difference has been observed between our samples and those purchased from Alfa. Annealing of $\mathrm{TiO}$ pellets at $1100{ }^{\circ} \mathrm{C}$ for $24 \mathrm{~h}$ under vacuum and slow

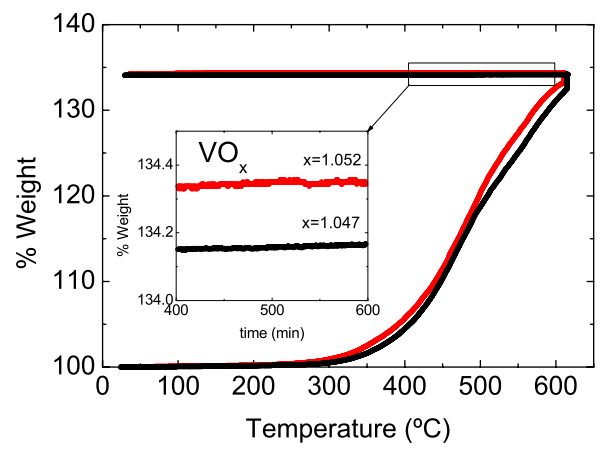

FIG. 2. (Color online) Typical weight gain during a thermogravimetric analysis experiment to determine the oxygen content. Accuracy of this method is better than 0.01 , as it can be observed in the inset.

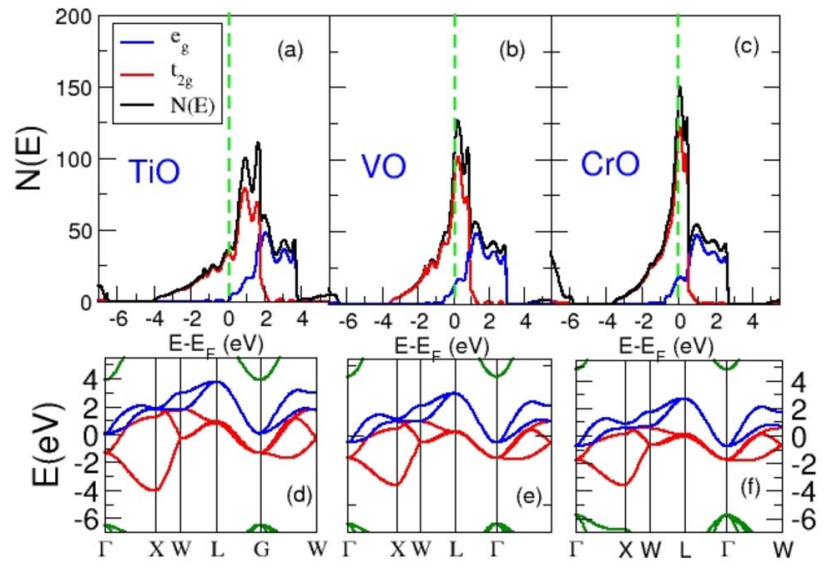

FIG. 3. (Color online) Electronic structure of TiO (left), VO (middle), and $\mathrm{CrO}$ (right) with density functional calculations in the gradient corrected approximation. In panels (a)-(c), we show the total density of states as well as the projections to the $t_{2 g}$ and $e_{g}$ levels (red and blue lines, respectively). The vertical line shows the Fermi energy. In panels (d)-(f), we show the bands closer to the Fermi energy. The $d t_{2 g}$ and $e_{g}$ bands are well separated from the $s p$ bands.

cooling (holding the sample at $900{ }^{\circ} \mathrm{C}$ for at least $48 \mathrm{~h}$ ) results in a monoclinic phase (space group $A 2 / \mathrm{m}$ ) with an ordered array of vacant lattice sites: half of the Ti and half of the $\mathrm{O}$ atoms are missing alternately in every third (110) plane. This process is completely reversible, and both ordered or disordered samples are stoichiometrically identical, within the error. All attempts to order the vacancies in the case of $\mathrm{VO}_{x}$ were unsuccessful.

\section{RESULTS AND DISCUSSION}

Figures 3(a)-3(f) show the energy bands and density of states of $\mathrm{TiO}, \mathrm{VO}$, and hypothetical $\mathrm{CrO}$ calculated with a gradient corrected local density approximation and localized atomic orbitals with CRYSTAL03. ${ }^{16}$ The calculations shown in the figure assume spin-unpolarized solutions and the lattice parameter that minimizes the ground state energy: 4.21, 4.16, and $4.11 \AA$ for $\mathrm{TiO}, \mathrm{VO}$, and $\mathrm{CrO}$, respectively. Under these approximations, the electronic structure of the three compounds is similar. They are metallic, with the $d$ bands well separated from the $s$ bands. In the case of $\mathrm{TiO}$, the Fermi energy lies well in the $t_{2 g}$ bands, which in all the three compounds overlap with the $e_{g}$ bands. Moving from $\mathrm{TiO}$ to $\mathrm{CrO}$, the bandwidth decreases, and the Fermi energy moves upwards, to accommodate electrons in states with some weight in the $e_{g}$ bands. Similar results are obtained using either B3LYP hybrid density functional ${ }^{17}$ or the $G W$ approach. ${ }^{18}$

The first experimental evidence of unconventional metallic behavior in VO comes from magnetic susceptibility and specific heat measurements of both $\mathrm{VO}_{x}$ and $\mathrm{TiO}_{1.0}$, with and without ordered vacancies. For $\mathrm{TiO}_{1.0}$, a temperature independent $\chi(T)$ (Fig. 4) and the asymptotic evolution of $C(T) / T$ toward a constant low-temperature value (inset of Fig. 4) are nicely consistent with the expectations of a stan- 


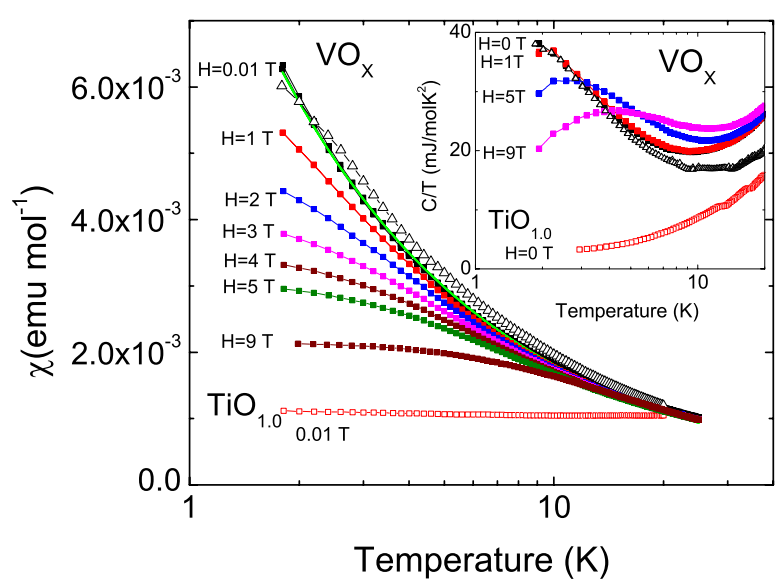

FIG. 4. (Color online) Low-temperature susceptibility of $\mathrm{VO}_{x}$ and $\mathrm{TiO}_{1.0}$ at different fields. Closed squares correspond to $x$ $=0.95$ at different fields, while open triangles refer to $x=0.975$ at $H=0.01 \mathrm{~T}$. The green line is a fit to $\left(\chi-\chi_{0}\right) \propto T^{-0.78}$. Inset: $C / T$ versus $T$ for the same samples at different magnetic fields. Open triangles correspond to $x=0.975(H=0)$ and closed symbols to $x$ $=0.95$ measured at different fields.

dard Fermi liquid, independent of disorder. In contrast, the susceptibility of $\mathrm{VO}_{x}$ is strongly enhanced upon cooling below $\approx 20 \mathrm{~K}$ and follows a power law $\left(\chi-\chi_{0}\right) \approx T^{-\eta}$, with $\chi_{0} \approx 2.7 \times 10^{-4} \mathrm{emu} \mathrm{mol}^{-1} \mathrm{Oe}^{-1}$ and $\eta \approx 0.78$ below $\approx 20 \mathrm{~K}$ (Fig. 4); a system of independent spins would obey a Curie law, with $\chi_{0} \approx 0$ and $\eta \approx 1$. Application of a magnetic field flattens the $\chi(T)$ curve, consistent with the opening of a Zeeman spin gap and the subsequent depletion of $\chi(T)$.

Specific heat at low temperature in $\mathrm{VO}_{0.95}$ also deviates from the linear temperature dependence and shows a strong magnetic field dependence (inset of Fig. 4) in both cases, in conflict with Fermi-liquid theory. $C(T) / T$ in $\mathrm{VO}_{x}$ follows a power law $\propto T^{-\eta}, \eta \approx 0.65$, below $\approx 10 \mathrm{~K}$. Moreover, a departure from this behavior is evident below $\approx 2 \mathrm{~K}$, due to the existence of a broad maximum, which becomes evident after application of a magnetic field.

The results shown in Fig. 4 correspond to two different compositions of $\mathrm{VO}_{x}(x=0.95$ and $x=0.975)$ and show that we have not detected a correlation between $x$ and the deviation from FL behavior. The behavior of the magnetic susceptibility and the specific heat in $\mathrm{VO}_{x}$ is absolutely unexpected and, at first sight, reminiscent of a spin glass above the freezing temperature. This would imply the presence of (interacting) localized spins in the FL, which is, in principle, unexpected for a conventional metal. In fact, a magnetic phase transition at $T=0$ plus disorder could lead to the observed spin-glass-like features in the susceptibility and specific heat. ${ }^{19}$ So departure of the susceptibility and specific heat from the standard behavior could be due to the tendency of $d$ electrons to form local spin moments in VO, resulting in some kind of collective relaxation state. ${ }^{20}$ The possibility of field tuning the properties of this system makes it very interesting from an experimental point of view. Following this argument, the collective state reminiscent of a spin glass would be an effect of the underlying electronic mechanism that produces the observed non-FL behavior.

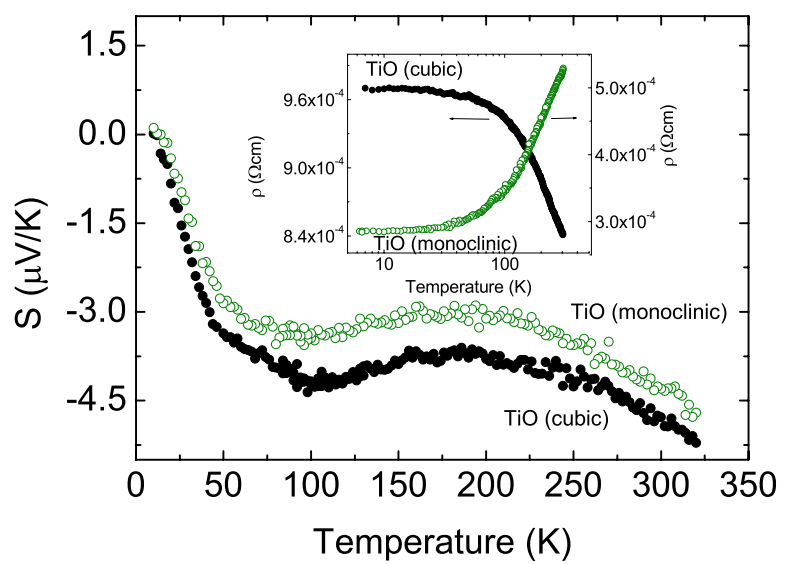

FIG. 5. (Color online) Thermoelectric power of $\mathrm{TiO}_{1.0}$ with the vacancies ordered (monoclinic) and disordered (cubic). The small difference in the absolute value $(\leqslant 0.5 \mu \mathrm{V} / \mathrm{K})$ must be due to a small variation in the stoichiometry produced by the annealing. In any case, the difference is irrelevant and the temperature dependence is clearly not affected by the ordering of the vacancies. Inset: effect of vacancy ordering on the resistivity of $\mathrm{TiO}_{1.0}$.

A priori, the origin of local magnetic moments and hence the failure of standard band theory to describe VO could be due either to disorder or to strong electronic correlations due to $W \approx U$. Disorder is certainly present in these compounds, which show a large number of vacancies at both the metal and oxygen sites. However, TiO has the same amount of vacancies but does not show any signature compatible with the presence of local spin moments. On the other hand, we have not observed any correlation between the number of vacancies present at different compositions and the apparent divergence of the $\chi(T)$ and $C(T) / T$, as can be seen from the comparison of two different compositions in Fig. 4.

Charged elementary excitations are probed in transport experiments. In the inset of Fig. 5, we show resistivity versus temperature $\rho(T)$ for the same sample of TiO before and after ordering the vacancies. It is apparent that the disordered cubic crystal behaves like a semiconductor, whereas the ordered monoclinic crystal has a metalliclike conductivity with a large temperature independent part due to vacancy scattering. Therefore, $\rho(T)$ curves are dominated by disorder and do not provide straightforward information about the effect of interactions on the quasiparticle dynamics.

Then, an observed $d \rho / d T<0$ in $\mathrm{VO}_{x}$ is not intrinsic but dominated by vacancy scattering. In contrast, we observe that thermoelectric power in $\mathrm{TiO}$ is not sensitive to disorder (Fig. 5). At low temperature, the phonon-drag enhancement dominates over the contribution from conventional electronic diffusion. Close to room temperature, the electronic contribution of itinerant charge carriers to the thermoelectric power, both in the case of conventional ${ }^{21}$ and strongly correlated metals, ${ }^{22}$ is given by

$$
S=-C \frac{k_{B}}{e}\left(\left.\frac{k_{B} T}{Z} \frac{d \ln \Phi(E)}{d E}\right|_{E=E_{F}}\right),
$$

where $C$ is a dimensionless constant, $e$ is the charge of the electron, $k_{B}$ is the Boltzmann constant, $T$ is the temperature, 


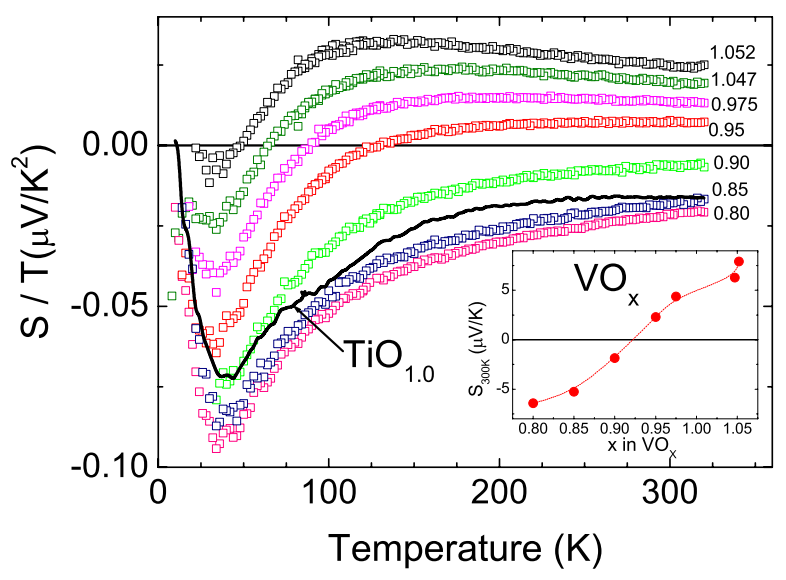

FIG. 6. (Color online) Thermoelectric power divided by temperature as a function of temperature. According to expression (1), the plot should be a constant value for a metal at high enough temperature. At low temperatures, the phonon-drag enhancement deviates the experimental values from the diffusion formula. Inset: variation of the thermopower at $300 \mathrm{~K}$ with $x$ in $\mathrm{VO}_{x}$. The change of sign occurs at $x \sim 0.95$, consistent with $\mathrm{x}$-ray absorption spectroscopy results (Ref. 23), which show a valence change of $(2-\delta)^{+}$ $\rightarrow(2+\delta)^{+}$at $x \sim 0.94-0.97$.

and $\Phi(E)=(1 / V) \Sigma_{k}\left(\partial \varepsilon_{k} / \partial k_{x}\right)^{2} \delta\left(E-\varepsilon_{k}\right)$ is a transport function with an energy dependence similar to that of the density of states $N(E)$. The thermoelectric power of a metal expressed by Eq. (1) has an intrinsic sign, which reflects the curvature of $N(E)$ around $E_{F}$. The negative $S(T)$ in $\mathrm{TiO}_{x}$ irrespective of $x$ (Ref. 13) is consistent with a one-third filled $\pi^{*}$ band, as obtained in the calculations. In contrast, the gradual change in the sign of $S(T)$ with $x$ in $\mathrm{VO}_{x}$ (see Fig. 6) signals a change in the curvature of the density of states around $E_{F}$, as it crosses the midband energy, which is at odds with Eq. (1). In fact, the change in the sign of $S(T)$ reported in Fig. 6 is compatible with a depression in the density of states (a pseudogap) around the Fermi energy in $\mathrm{VO}_{x}$.

Having more than one band that crosses the Fermi energy should not change this interpretation of the thermopower, as the dominant mechanism will be the scattering of charge carriers from the wider to the narrower bands (due to the higher density of states), introducing a scattering rate proportional to $N(E)$. As a result, the thermopower will present a term which is proportional to the derivative of the density of states of the narrower band with respect to the energy at $E_{F}$.

It is important to note that the change in the chemical potential necessary to account for the electron density difference between $\mathrm{VO}_{0.9}$ and $\mathrm{VO}_{1.1}$ is much smaller than the typical energy scales in which the $a b$ initio density of states varies. Therefore, the change of sign of the thermopower is due to dynamic electronic correlations absent in density functional theory calculations. In particular, short-range spin correlations in a doped Mott insulator can give rise to a pseudogap in the density of states. ${ }^{24}$ Note that although the characteristic energy scale of the non-Fermi-liquid phenomena should be much smaller than that of the pseudogap formation, we have not found any systematic variation of the magnitude of this behavior (magnitude of the magnetic mo- ment, etc.) with $x$. All of the above is compatible with the opening of a pseudogap close to the Fermi energy due to dynamic spin fluctuations, although direct spectroscopic experiments should confirm or reject this scenario. The same correlation effects might result in the opening of the pseudogap in the quasiparticle spectrum and in the anomalous collective spin modes responsible for the unexpected dependence of the specific heat and susceptibility on the magnetic field. Whereas the quasiparticle density of states at the Fermi energy seems to show a variation as a function of composition, the collective spin modes depend less on the composition $x$.

Another indication of the connection between the anomalous magnetic and transport properties of $\mathrm{VO}_{x}$ comes from the high positive magnetoresistance observed by Rata et al. ${ }^{25}$ One possibility against the correlation-driven pseudogap is the appearance of a mobility gap due to Anderson localization, which could give rise to local moments due to disorder. Random distribution of both $\mathrm{V}^{2+}$ and $\mathrm{O}^{2-}$ vacancies in $\mathrm{VO}_{x}$ introduces a variation in the periodic potential from site to site that could localize the electronic wave functions if strong enough. This is expected to occur above a critical value of the ratio between the random potential and the bandwidth. From the band structure calculations performed in Fig. 3, the difference in the bandwidth between $\mathrm{TiO}$ and $\mathrm{VO}$ is very small so that the effect of disorder in both materials must be very similar. The negligible effect in TiO almost completely rules out this explanation. Note that the opposite interpretation, i.e., a stronger effect of vacancies in VO with respect to $\mathrm{TiO}$ will point to a much different and unexpected electronic state between these two compounds.

However, to discard fully Anderson localization, we have synthesized samples of $\mathrm{VO}_{x}$ approaching the limit of solubility of the system $(x \approx 0.2)$ and measured their thermopower. As $x$ increases in $\mathrm{VO}_{x}$, the number of $\mathrm{V}$ vacancies increases and, hence, the perturbation of the periodic potential experienced by the conduction electrons. For samples with a large amount of $\mathrm{V}$ vacancies, the thermopower deviates from the Mott formula and shows clear signs of activated behavior (Fig. 7). However, disordered vacancies in VO do not set $E_{F}$ below a mobility edge for the range of $0.8<x<1.1$ studied in this work.

Our experimental results demonstrate that the low-energy elementary excitation spectrum of VO is dominated by some kind of spin fluctuation without long-range order. The tendency of VO to develop local magnetic moments is supported by our spin-polarized density functional calculations. For $\mathrm{VO}$ and $\mathrm{CrO}$, either ferromagnetic or antiferromagnetic solutions are much lower in energy than the paramagnetic one, both within the generalized gradient approximation (GGA) and B3LYP functionals. In contrast, the paramagnetic electronic structure of $\mathrm{TiO}$ calculated with the GGA functional has a smaller energy than the spin-polarized solutions, in agreement with the experiment. This is another indication that whereas $\mathrm{TiO}$ is a band conductor, $\mathrm{VO}$ and $\mathrm{CrO}$ have a tendency to develop local moments with spin $3 / 2$ and $4 / 2$, respectively.

The development of local moments would have consequences on the stability of the lattice. According to the electronic structure calculations, the equilibrium lattice constant 


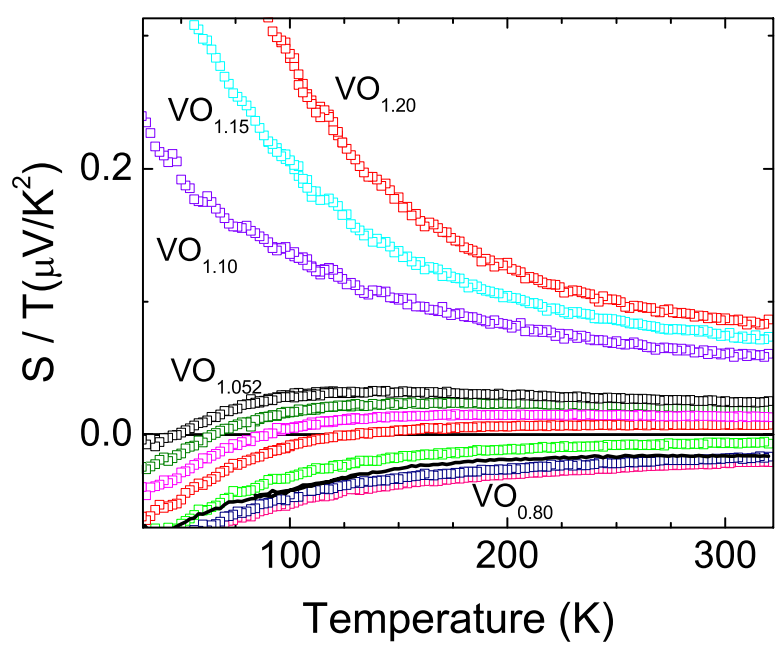

FIG. 7. (Color online) Themoelectric power versus temperature for samples with a large amount of vacancies. Large $(x>1.1)$ random quenched disorder introduces localized states and departure of intrinsic metallic behavior.

of VO is 4.3 for spin-polarized solutions and 4.16 for spinunpolarized ones. Thereby, the spin fluctuations revealed by our experiments would be accompanied by strong lattice fluctuations. This scenario of bond-length fluctuations corroborates that proposed earlier ${ }^{26}$ to account for the suppression of the phonon contribution to the thermal conductivity and of vacancy ordering. This spin-lattice coupling is also obtained in our calculations for $\mathrm{CrO}$. In this case, the occupation of the orbitals depends on the spin, whereas paramagnetic $\mathrm{CrO}$ accommodates four electrons mostly in the $t_{2 g}$ bands; our density functional calculations, both with GGA and B3LYP, show that spin-polarized $\mathrm{CrO}$ is high spin $(4 / 2)$ and thereby one electron occupies the doubly degenerate $e_{g}$ band. Therefore, because of the local moment formation, the fourth electron in $\mathrm{CrO}$ goes into an $e_{g}$ state. This strong magnetoelastic effect is expected to be accompanied by large fluctuations of the local charge distribution, ${ }^{27}$ setting the $\mathrm{CrO}$ system in an unstable situation against a spontaneous charge disproportionation reaction. In fact, all attempts to synthesize $\mathrm{CrO}$ finished with $\mathrm{Cr}+\mathrm{Cr}_{2} \mathrm{O}_{3}$. Therefore, we propose that the inaccessibility of $\mathrm{CrO}$ at ambient pressure might be related to correlation-driven electronic phase segregation. ${ }^{28}$

Our main findings are incorporated in a phase diagram for the monoxides of the first transition series, presented in Fig. 8. The $T_{C}$ for $\mathrm{TiO}_{x}$ is from Ref. 12. We claim that the origin of the behavior of $\mathrm{VO}$ is related to the tendency of the $d$ electrons to form local spin moments in the vicinity of a metal-insulator transition. From the insulating side, this transition has been recently observed in $\mathrm{MnO}$ under hydrostatic pressure ${ }^{29}$ which changes the $U / W$ ratio, keeping the number of electrons constant. Our data on VO shed light on the behavior of the metallic side approaching the localized limit

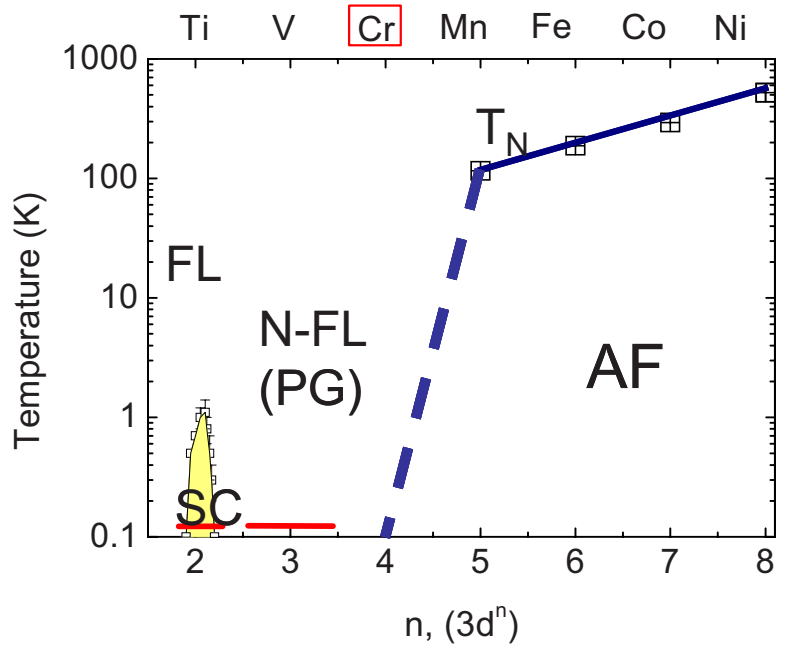

FIG. 8. (Color online) Unified electronic phase diagram of the monoxides of the first transition series. It does represent a generic electronic or magnetic phase diagram of a cubic (rocksalt) structure, in which $3 d$ electrons are successively added into it. In spite of the simplicity of the structure and its three dimensional character, the diagram shows many of the peculiarities of the diagram of more complex systems. The red horizontal bars indicate the range of doping explored in $\mathrm{TiO}_{x}$ and $\mathrm{VO}_{x}$. $\mathrm{Cr}$ is squared to signal that bulk $\mathrm{CrO}$ does not exist. The dotted line represents the uncertainty to locate precisely the quantum phase transition. FL stands for Fermi liquid; N-FL, non-Fermi-liquid; PG, pseudogap; SC, superconductor; AF, antiferromagnet; and $T_{N}$ is the Neel temperature.

upon doping. Interestingly, the compound at which the metal-insulator transition is expected, $\mathrm{CrO}$ is not stable.

In summary, based upon careful thermodynamic and transport experiments on a variety of $\mathrm{VO}_{x}$ and $\mathrm{TiO}$ samples, we have presented a global picture of the $3 d$ transition-metal monoxides and their metal-insulator transition. We have presented compelling evidence to claim that, in spite of its simple chemical and crystallographic structure, VO is a correlated metal with an exotic electronic phenomenology, similar to other strongly correlated systems. We also hope that our results will stimulate an experimental confirmation of the pseudogap by direct spectroscopic measurements.

\section{ACKNOWLEDGMENTS}

M. C. Aronson, G. Kotliar, D. Khomskii, L. H. Tjeng, S. S. Saxena, and L. E. Hueso are acknowledged by discussion and critical reading of the paper. H.-D. Zhou is acknowledged by assistance during transport measurements. We are also grateful for financial support from Xunta de Galicia (Project No. PXIB20919PR), the Ministry of Science of Spain (MAT2004-05130-C02-01 and MAT2005-06024-C0201), and Program Ramón y Cajal (F.R.). 
*qffran@usc.es

${ }^{1}$ N. W. Aschcroft and N. D. Mermin, in Solid State Physics (Holt, Rinehart and Winston, New York, 1976).

${ }^{2}$ J. Zaanen, G. A. Sawatzky, and J. W. Allen, Phys. Rev. Lett. 55, 418 (1985).

${ }^{3}$ H. v. Löhneysen, T. Pietrus, G. Portisch, H. G. Schlager, A. Schröder, M. Sieck, and T. Trappmann, Phys. Rev. Lett. 72, 3262 (1994).

${ }^{4}$ M. C. Aronson, R. Osborn, R. A. Robinson, J. W. Lynn, R. Chau, C. L. Seaman, and M. B. Maple, Phys. Rev. Lett. 75, 725 (1995).

${ }^{5}$ R. W. Hill, C. Proust, L. Taillefer, P. Fournier, and R. L. Greene, Nature (London) 414, 711 (2001).

${ }^{6}$ J.-S. Zhou, W. Archibald, and J. B. Goodenough, Nature (London) 381, 770 (1996).

${ }^{7}$ A. Yeh, Y.-A. Soh, J. Brooke, G. Aepli, T. F. Rosenbaum, and S. M. Hayden, Nature (London) 419, 459 (2002).

${ }^{8}$ C. Pfleiderer, S. R. Julian, and G. G. Lonzarich, Nature (London) 414, 427 (2001).

${ }^{9}$ H. Ding, T. Yokoya, J. C. Campuzano, T. Takahashi, M. Randeria, M. R. Norman, T. Mochiku, K. Kadowaki, and J. Giapintzakis, Nature (London) 382, 51 (1996).

${ }^{10}$ D. S. Dessau, T. Saitoh, C.-H. Park, Z.-X. Shen, P. Villella, N. Hamada, Y. Moritomo, and Y. Tokura, Phys. Rev. Lett. 81, 192 (1998).

${ }^{11}$ A. Moreo, M. Mayr, A. Feiguin, S. Yunoki, and E. Dagotto, Phys. Rev. Lett. 84, 5568 (2000)

${ }^{12}$ J. K. Hulm, C. K. Jones, R. A. Hein, and J. W. Gibson, J. Low Temp. Phys. 7, 291 (1972).

${ }^{13}$ M. D. Banus, T. B. Reed, and A. J. Strauss, Phys. Rev. B 5, 2775 (1972).

${ }^{14}$ J. B. Goodenough, Phys. Rev. B 5, 2764 (1972).
${ }^{15}$ F. J. Morin, Phys. Rev. Lett. 3, 34 (1959).

${ }^{16}$ V. R. Saunders, R. Dovesi, C. Roetti, M. Causà, N. M. Harrison, R. Orlando, and C. M. Zicovich-Wilson, CRYSTAL98 User's Manual (University of Torino, Torino, 1998).

${ }^{17}$ W. C. Mackrodt, D. S. Middlemiss, and T. G. Owens, Phys. Rev. B 69, 115119 (2004).

${ }^{18}$ A. Yamasaki and T. Fujiwara, Phys. Rev. B 66, 245108 (2002).

${ }^{19}$ A. H. Castro Neto, G. Castilla, and B. A. Jones, Phys. Rev. Lett. 81, 3531 (1998).

${ }^{20}$ D. A. Gajewski, N. R. Dilley, R. Chau, and M. B. Maple, J. Phys.: Condens. Matter 8, 9793 (1996).

${ }^{21}$ N. F. Mott and H. Jones, in The Theory of the Properties of Metals and Alloys (Dover, New York, 1936).

${ }^{22}$ G. Palsson and G. Kotliar, Phys. Rev. Lett. 80, 4775 (1998).

${ }^{23}$ A. D. Rata, A. R. Chezan, M. W. Haverkort, H. H. Hsieh, H.-J. Lin, C. T. Chen, L. H. Tjeng, and T. Hibma, Phys. Rev. B 69 , 075404 (2004).

${ }^{24}$ B. Kyung, S. S. Kancharla, D. Sénéchal, A.-M. S. Tremblay, M. Civelli, and G. Kotliar, Phys. Rev. B 73, 165114 (2006).

${ }^{25}$ A. D. Rata, V. Kataev, D. Khomskii, and T. Hibma, Phys. Rev. B 68, 220403(R) (2003).

${ }^{26}$ J. B. Goodenough, F. Rivadulla, E. Winkler, and J.-S. Zhou, Europhys. Lett. 61, 527 (2003).

${ }^{27}$ J. Arvanitidis, K. Papagelis, S. Margadonna, K. Prassides, and A. N. Fitch, Nature (London) 425, 599 (2003).

${ }^{28}$ R. Jamei, S. Kivelson, and B. Spivak, Phys. Rev. Lett. 94, 056805 (2005).

${ }^{29}$ C. S. Yoo, B. Maddox, J. H. P. Klepeis, V. Iota, W. Evans, A. McMahan, M. Y. Hu, P. Chow, M. Somayazulu, D. Häusermann, R. T. Scalettar, and W. E. Pickett, Phys. Rev. Lett. 94, 115502 (2005). 